\title{
TagFall: Towards Unobstructive Fine-Grained Fall Detection based on UHF Passive RFID Tags
}

\author{
Wenjie Ruan, Lina Yao, \\ Quan Z. Sheng, Nickolas \\ J.G. Falkner \\ School of Computer Science \\ The University of Adelaide \\ Adelaide, SA 5005, Australia \\ \{wenjie.ruan; lina.yao\}@adelaide.edu.au \\ \{qsheng;jnick\}@cs.adelaide.edu.au \\ Xue Li \\ School of ITEE \\ The University of Queensland \\ Brisbane, QLD 4072, Australia \\ xueli@itee.uq.edu.au
}

\author{
Tao Gu \\ School of CS\&IT \\ RMIT University \\ Melbourne, VIC 3000, \\ Australia \\ tao.gu@rmit.edu.au
}

\begin{abstract}
Falls are among the leading causes of hospitalization for the elderly and illness individuals. Considering that the elderly often live alone and receive only irregular visits, it is essential to develop such a system that can effectively detect a fall or abnormal activities. However, previous fall detection systems either require to wear sensors or are able to detect a fall but fail to provide fine-grained contextual information (e.g., what is the person doing before falling, falling directions). In this paper, we propose a device-free, fine-grained fall detection system based on pure passive Ultra-High Frequency (UHF) Radio-Frequency IDentification (RFID) tags, which not only is capable of sensing regular actions and fall events simultaneously, but also provide caregivers the contexts of fall orientations. We first augment the Angle-based Outlier Detection Method (ABOD) to classify normal actions (e.g., standing, sitting, lying and walking) and detect a fall event. Once a fall event is detected, we first segment a fix-length RSSI data stream generated by the fall and then utilize Dynamic Time Warping (DTW) based $k$ Nearest Neighbors $(k \mathrm{NN})$ to distinguish the falling direction. The experimental results demonstrate that our proposed approach can distinguish the living status before fall happening, as well as the fall orientations with a high accuracy. The experiments also show that our device-free, fine-grained fall detection system offers a good overall performance and has the potential to better support the assisted living of older people.
\end{abstract}

\section{Categories and Subject Descriptors}

C.4 [Special-Purpose and Application-Based System ]: Realtime and RFID-based system

\section{Keywords}

Fall Detection, Device-Free, RFID, Anomaly Detection, House- hold Monitoring

\section{INTRODUCTION}

Falls happen when human body suddenly changes from a normal living status (e.g., sitting, standing, or walking) to the reclining without control [25], which often occur in a very short time without human attentions. Falls may cause moderate to severe injuries including hip fractures, head traumas, even more devastating consequences for the elderly. Based on the Centers for Disease Control and Prevention, one-third population of the elderly who aged 65 and older experience falls each year [32]. Researchers estimate that up to $50 \%$ of nursing home residents fall each year and more than $40 \%$ of them might fall more than once [32]. Moreover, studies have shown that the medical outcome of a fall is largely dependent on the response and rescue time[16]. The delay of medical treatment after a fall can increase the mortality risk in some clinical conditions, especially for those who live alone [29]. Thus, falls are a major health risk that diminishes the quality of life among the elderly people, strongly motivating the necessity of fall detection systems.
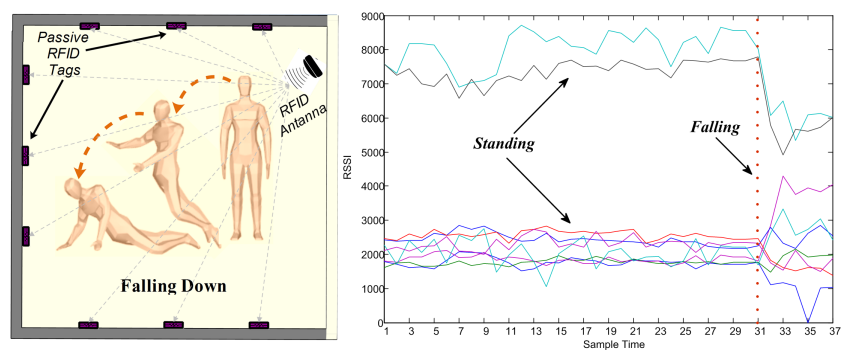

Figure 1: RSSIs variation patterns when falls occur

Over the past decades, fall detection (FD) and prevention have been an active research area with several proposed solutions. Both wearable sensor based (e.g., inertial sensors [2], accelerometer $[3,8]$, specialized cane [16]) and smart-phone based $[5,14]$ fall detection techniques require the subject to be attached with sensors or phones, which might not be practical (e.g., sensors lost/damaged, or forget to carry by the elderly with dementia). Vision based fall detection systems $[9,27,18]$ employ activity classification algorithms on a series of images recorded by a video camera, which is usually regarded as being privacy invasive and causes uncomfortable 
feeling to the elderly. Vision-based systems also fail to work in dimmed or dark environments, where falls usually happen.

Recently, some device-free techniques for fall detection have been proposed [26, 20,31]. However, in most of these systems, some complicated or personalized devices (e.g., pressure sensor, audio sensor, radar) are needed to be implanted in the environment, and then the variations of audio, pressure or microwave signals are used to infer a fall event. As a result, most of them can only sense whether a fall happened, but fail to provide more fine-grained information [23], which is valuable to caregivers. One of the fine-grained contexts is the status (e.g., sitting, standing or walking) before a fall occurs. For example, when people fall down while standing or siting, some serious diseases possibly have happened such as cerebral haemorrhage or cardiopathy [32]. But when people are walking, the falling is possibly caused by knocking some obstacles. Another useful contextual information is the fall orientations (e.g., fall to front, fall to back or fall to the right side), such as falls to back may seriously damage the subject's head, while falling to the right side may more likely cause injuries to arms or legs.

Based on these motivations and the prosperous development of RFID techniques, this paper aims to investigate if device-free, fine-grained fall detection can be achieved without any carried devices by using pure UHF passive RFID tags. Figure 1 illustrates the basic intuitions of our work. When the subject falls from standing, the Received Signal Strength Indicators (RSSIs) show different fluctuation patterns, indicating the potential for detecting a fall. Compared to other hardware platforms, RFID is cost-effective (passive tags cost several cents each) and practical (e.g., no maintenance since no battery needed) [28]. Moreover, there are few works so far studying the relationship between RSSIs variations of passive tags and human activities (e.g., falls).

In this paper, we propose an unobstructive, fine-grained fall detection system called TagFall, which can detect a fall event, be capable to sense normal living actions (e.g., standing, sitting, lying and walking in our case), and can distinguish the falling directions. To achieve a fine-grained fall detection, our TagFall mainly consists of two detection phases: a) Detecting Normal Actions and Falls: we augment Angle-based Outlier Detection (ABOD) [15] method to mine the clustering patterns of RSSIs (generating by normal human actions) and detect an anomaly pattern (caused by falls) simultaneously; and b) Detecting Fall Directions: once we detect a fall happened, we segment a fix-length data stream, which we use to calculate the Dynamic Time Warping (DTW) [11] distance with profiled data streams (known labels). So we can distinguish the fall directions by a majority vote of its $k$ nearest neighbors based on the DTW distances. In summary, the core idea of this paper is to mine the clustering patterns and change rules of RSSIs when the environment is affected by different human actions (e.g., normal activities and falls with different orientations). The main contributions are listed as follows.

- We exploit the feasibility of using passive RFID tags to achieve unobstructive, fine-grained fall detection. To the best of our knowledge, this is the first work to leverage RSSI signals for device-free fall detection based on pure passive RFID tags.

- We propose a fine-grained fall detection pipeline, which

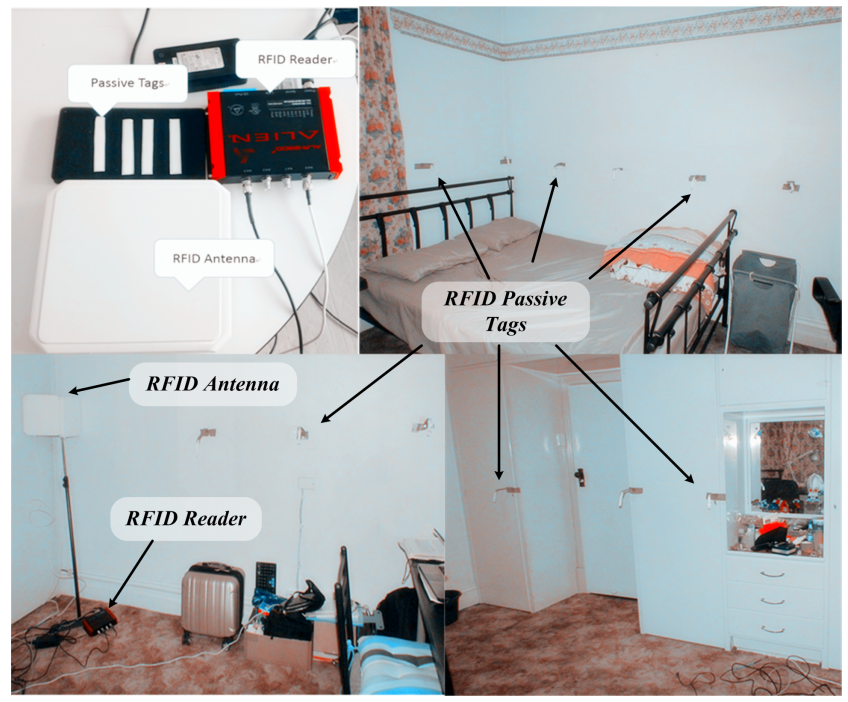

Figure 2: Hardware Deployment

not only can detect a fall event, but also be capable of offering the contextual information of the subject's status before falls occur and the falling directions.

The rest of the paper is organized as follows. Section 2 introduces the hardwares and intuitions of our system. We present our system architecture in Section 3 and propose our solutions in Section 4. Section 5 presents experimental results and analysis. Section 6 reviews related work and Section 7 gives the discussion. Finally, Section 8 offers some concluding remarks.

\section{HARDWARE AND INTUITIONS}

Figure 2 shows the system setup, including an Alien ALR$9900+$ Enterprise RFID Reader $(20.3 \mathrm{~cm} \times 17.8 \mathrm{~cm} \times 4.1 \mathrm{~cm})$, two-circular antennas $(20 \mathrm{~cm} \times 20 \mathrm{~cm} \times 3 \mathrm{~cm})$, and squiggle Higgs- 4 passive tags $(1 \mathrm{~cm} \times 10 \mathrm{~cm})$. The reader operates at $840-960 \mathrm{MHz}$ and supports UHF RFID standards such as ETSI EN 302 208-1. We set the sample rate as $0.5 s$ and each tag reading contains a time-stamp, a tag ID, an antenna ID and an RSSI value, which is processed by a WINDOWS 7 $\mathrm{PC}$ with an I7-3537U $2.5 \mathrm{GHz}$ processor and $8 \mathrm{G}$ RAM.

Based on our preliminary experiments, we place the antenna $1.5 \mathrm{~m}$ above the ground, facing tags with approximately $60^{\circ}$, and attach tags on the wall with an approximate $0.6 \mathrm{~m}$ interval. When people perform activities in the testing area, an antenna can not guarantee to read all tags (interfered by human body), particularly for passive tags. To avoid this, we send an RSSI request to all tags within a sampling time. If we cannot receive RSSI readings of a certain tag, the RSSI value is manually set to 0 . Thus, mathematically, for all time stamps, we have the RSSI vectors with the same dimensions. In our settings, the tag detection range can be up to 6 meters. It is worth mentioning that, the electromagnetic fields generated by the readers, in any case, remain lower than the limitation of thresholding value for humans based on the report [22], which means the hardware used have no health risk to subjects.

Based on the hardware, we first run a series of pilot experiments to validate our intuitions. Figure 3 demonstrates that when the subject in different living status, the RSSIs display 


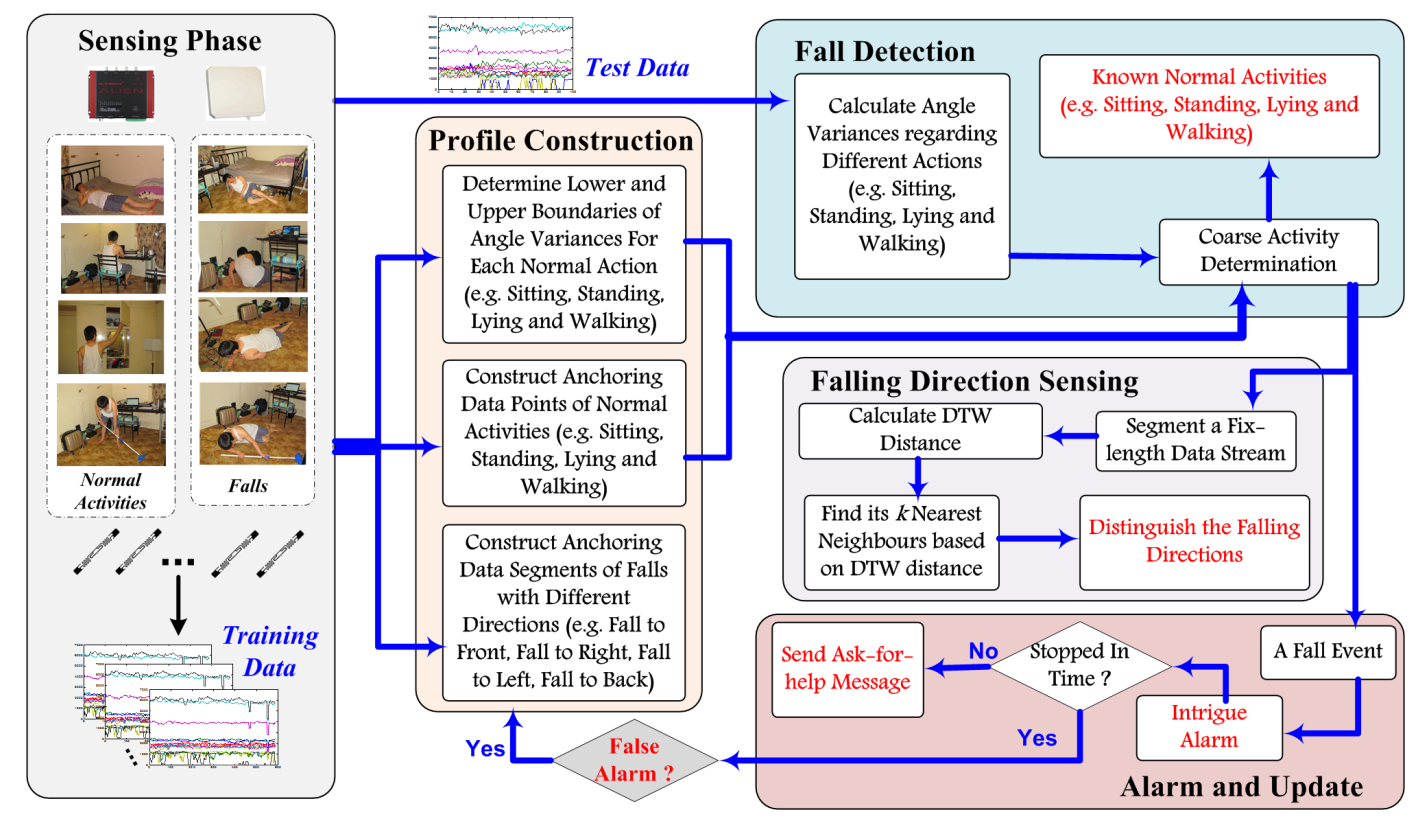

Figure 4: System Architecture

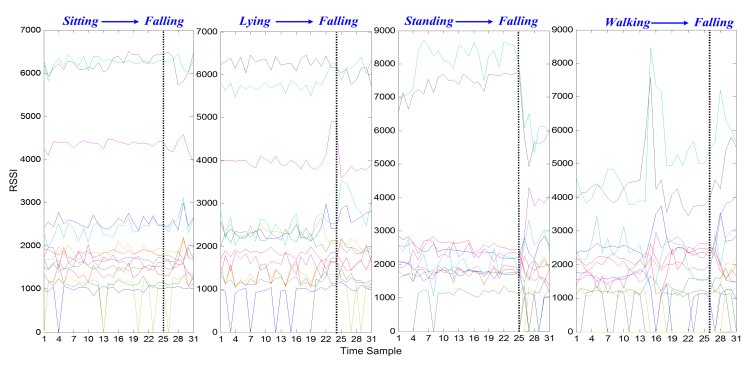

Figure 3: RSSIs variation patterns when a subject falls from different status

different invariant patterns. When the subject falls down from normal status (e.g., sitting, lying, standing or walking), the RSSIs reflect some unique variations that are different from previous stable patterns. Underpinned by these observations, it is possible for us to utilize some supervised classification algorithms to distinguish resident's regular living actions, as well as adopt anomaly detection method to detect an abnormal event (e.g., falls). In the next section, we will introduce how to tackle both problems by extending the traditional ABOD method.

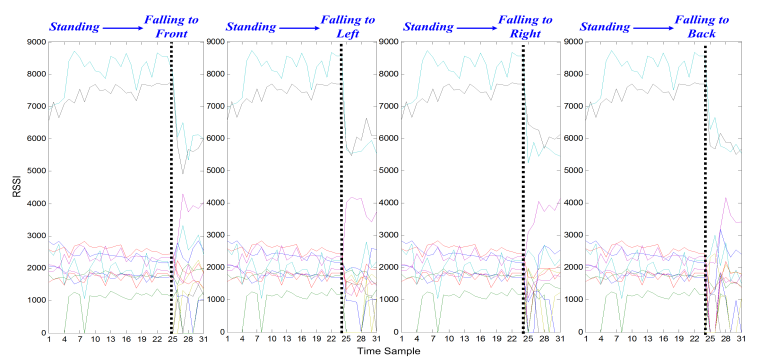

Figure 5: RSSIs variation patterns when a subject falls to different directions from standing
Figure 5 shows that the measured RSSIs can reveal varied fluctuation patterns due to the subject's falling down to different directions (e.g., front, left, right or back side) from standing, which allow us to utilize such underlying trends to recognize the falling orientations. Motivated by the observation, we could adopt data-stream classification methods to classify resident's falling directions. In this paper, we solve this problem by using DTW distance based $k \mathrm{NN}$. Overall, our preliminary studies have shown the feasibility and potential of our TagFall system to achieve a fine-grained fall detection.

\section{SYSTEM ARCHITECTURE}

Figure 4 shows an overview of proposed system. It consists of five main phases: the sensing phase, the profile construction phase, the fall detection phase, the falling direction sensing phase, and the altering phase.

Activity Sensing phase: the antenna in the test area collects RSSI readings propagated by passive tags and then sends them to the reader, which delivers the data package including RSSI values, time stamp, antenna ID and tag ID to a desktop computer for further processing.

Profile construction phase: we first utilize slide average smoothing to filter the noises caused by temperature, humidity changes [28] and categorize daily activities into four categories (i.e., sitting, standing, lying and walking, shown by Figure 12). Then, we calculate the angle variances of vector pairs formed by same action category and decide the upper and lower boundary of variances, which contain most likely variances. In the meantime, we sample the most representative data point for each regular action category to speed up the later online angle variance calculation. We also collect segmented data streams generated by falls with various falling directions to build the anchoring data streams for the later DTW distance calculations. 
Fall Detection phase: we perform the same smoothing as Profile Constriction phase and then calculate the angle variances of vector pairs formed by an observed RSSI and profiling data points for each normal action category. Based on the calculated variances (i.e., 4 variances in our case) and learned variance bounds, we identify the target's current actions by judging whether the variance lie in corresponding boundaries. If the variances are within the bounds of multiple action categories, we assign the activity label that has the most likely variance. When all four variances are beyond the bounds of known regular actions, the observed RSSI is regarded as an anomaly, which means the subject currently is experiencing a fall.

Falling Direction Sensing phase: once we detect a fall event, we first segment a data stream with the same length as the anchoring data streams. Then, we calculate the DTW distances between segmented data stream and all anchoring data streams. At last, we can distinguish the falling direction by a majority vote of its $k$ nearest neighbors regarding the DTW distance.

Altering and Update phase: in the meantime, we issue an alarm (e.g., ring an alarm bell) when a fall event is detected. If the user does not timely stop the alarm, we send an askfor-help SMS or call. Also, if the alarm is timely stopped but is a false alarm, we update the profiling data by adding the error-detected samples into right action category to enhance the detection performance.

\section{THE PROPOSED SOLUTION}

The key phases of our TagFall are how to efficiently distinguish the normal daily living actions and a fall event, and how to accurately classify the falling directions. In this section, we will introduce technical details on how these two problems are solved.

\subsection{Fall Detection}

One of the challenges in this paper is to detect the anomalous patterns in RSSI signals. A fall involves a series intensive posture changes (e.g., human postures sudden alter from standing, sitting or lying to the ground), which result in sudden, wide range fluctuation of RSSI patterns (see Figure 3 and 5 ). To tackle the challenge, we propose a $p$-partial Angle-based Outlier Detection that can identify $p$ categories of human regular actions and isolate the anomaly patterns. Angle-based Outlier Detection is first proposed by HansPeter Kriegel et al. [15] for finding anomalous data points caused by a different responsible mechanism. Unlike purely distance-based approaches (e.g., Local Outlier Factor [4]), ABOD does not rely on any parameter selection influencing the quality of achieved results. Here, we extend ABOD to do both classification and anomaly detection by mining the different patterns of angle variances paired by intra-action and inter-actions.

Figure 6 illustrates the basic intuition of our approach. For points (can be multi-dimension) generated by a same human activity, the angles between different vector pairs differ widely, which means a large angle variance (e.g., angle $\alpha_{1}, \alpha_{2}$, the variance of angle paired by data points of sitting is ranged from $1.17 \times 10^{-10} \sim 8.18 \times 10^{-6}$ ). The angle variance of vector pairs generated by different human activities is smaller since most points are clustered in some directions (e.g., angle $\beta_{1}, \beta_{2}$, the variance of angle paired
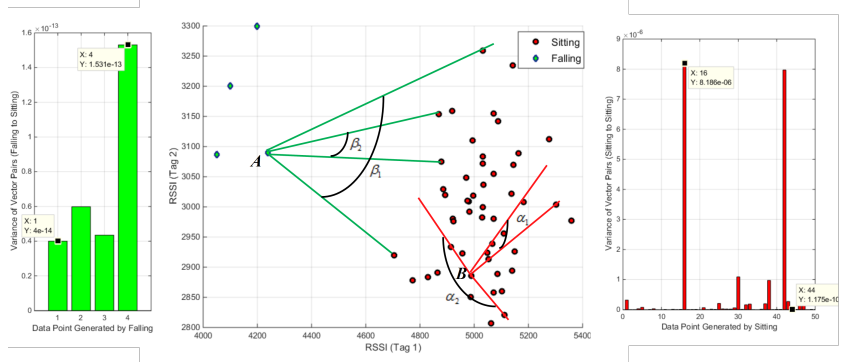

Figure 6: Intuition of angle-based outlier detection

from data points of falling to data points of sitting is ranged from $\left.4 \times 10^{-14} \sim 1.53 \times 10^{-13}\right)$. Therefore, we can classify different regular actions (easily collected, e.g., standing, sitting and walking), and detect abnormal actions (difficultly obtained, e.g., falling) by measuring the angle variances between a testing data point and the constructed profiling dataset. We first give the definition of Angle-based Outlier Factor (ABOF) [15] which measures the angle variance of a data point paired with other data points.

Definition 1 (ABOF). Given a database $\mathcal{D} \in \mathbb{R}^{d}$, a point $A \in \mathcal{D}$, and a norm \|\| . The scalar product is denoted by $<., .>$. For two points $B, C \in \mathcal{D}, \overline{B C}$ denotes the difference vector $C-B$. The angle-based outlier factor $A B O F(A)$ is the variance over the angles between the difference vectors of $A$ to all pairs in $D$ weighted by the distance of the points:

$$
\begin{aligned}
& A B O F(A)=V A R_{B, C \in \mathcal{D}}\left(\frac{<\overline{A B}, \overline{A C}>}{\|\overline{A B}\|^{2} \cdot\|\overline{A C}\|^{2}}\right) \\
& =\frac{\sum_{B \in \mathcal{D}} \sum_{C \in \mathcal{D}}\left(\frac{1}{\|\overline{A B}\| \cdot\|\overline{A C}\|} \cdot \frac{<\overline{A B}, \overline{A C}>}{\|\overline{A B}\|^{2} \cdot\|\overline{A C}\|^{2}}\right)^{2}}{\sum_{B \in \mathcal{D}} \sum_{C \in \mathcal{D}} \frac{1}{\|\overline{A B}\| \cdot\|\overline{A C}\|}} \\
& -\left(\frac{\sum_{B \in \mathcal{D}} \sum_{C \in \mathcal{D}} \frac{1}{\|\overline{A B}\| \cdot\|\overline{A C}\|} \cdot \frac{<\overline{A B}, \overline{A C}>}{\|\overline{A B}\|^{2} \cdot\|\overline{A C}\|^{2}}}{\sum_{B \in \mathcal{D}} \sum_{C \in \mathcal{D}} \frac{1}{\|\overline{A B}\| \cdot\|\overline{A C}\|}}\right)^{2}
\end{aligned}
$$

Based on ABOF, our proposed $p$-partial ABOD works as follows. Given that we already have profiling dataset regarding the resident's different regular living activities, we first compute off-line the angle variances of vector pairs in the dataset generated by the same activity for all $p$ categories ( $p=4$ in our case, i.e., sitting, standing, lying and walking, see Figure $7(\mathrm{a}) \sim(\mathrm{d}))$. Then, based on the variances, we decide the lower and upper bounds for the $p$ categories by a box and whisker diagram, which is a standardized way of displaying the distribution of data (see Figure 12). We then can online calculate the angle variances of an observed RSSI vector paired with the $p$-category profiling datasets (i.e., falling to sitting, falling to standing, falling to lying and falling to walking, see Figure 7 (e)). We assign labels to the test sample whose angle variances are within the corresponding boundaries. If multiple labels are assigned, we choose the category that the corresponding variance of testing data point lies in the middle of box as most (e.g., assume that the angle variances of a test data point paired with walking data and standing data are both $10^{-12}$, but the variance for walking data is in the middle of the box 


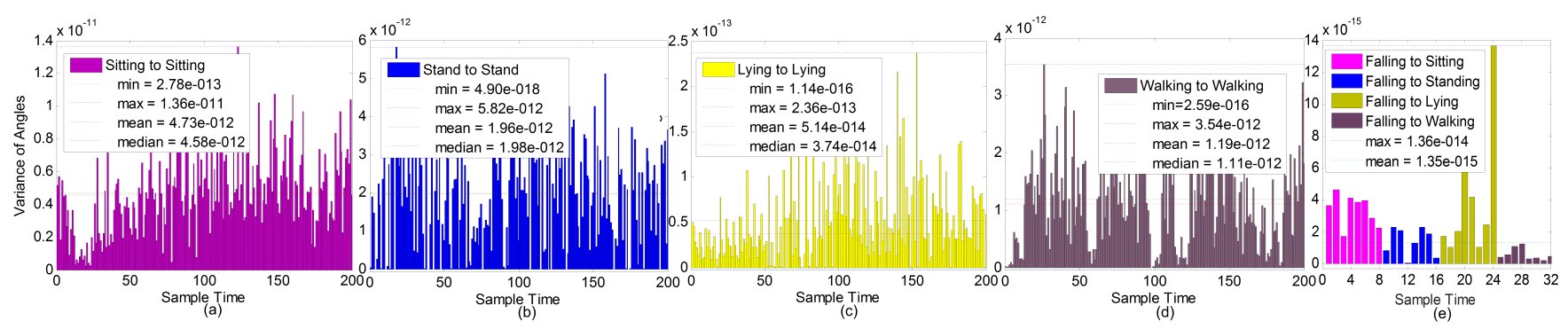

Figure 7: Intuition of $p$ ABOD
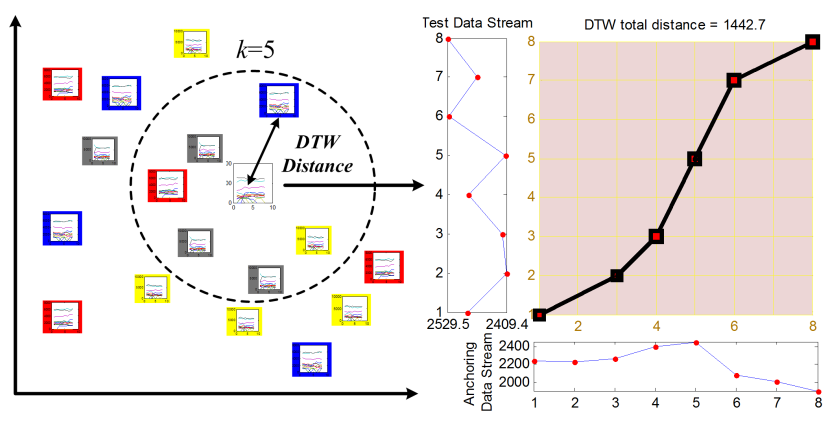

Figure 8: Outline of DTW based $k \mathrm{NN}$

more, see Figure 12, we assign the test data as walking). If no labels are assigned, we treat it as a potential outlier.

Unlike previous fall detection systems which usually either utilize some learning-based classification to distinguish a falling event from other activities [23], or first adopt anomaly detection method to detect an outlier point and then perform one-vs-all classification [12], our method focuses on mining the clustering patterns of RSSI data based on the intra angle-variance and inter angle-variance of multiple-group dataset to avoid parameter tuning.

\subsection{Falling Direction Sensing}

Sensing the falling direction in fact is a time-series classification problem. We need to classify the segmented RSSI data stream with a label (i.e., falling directions). To tackle the problem, we introduce a DTW based $k \mathrm{NN}$ method.

DTW is an efficient algorithm for measuring similarity between two temporal sequences which may vary in time or speed (e.g., walking pattern, speech recognition) [11]. Given two multi-dimensional time-series, $\mathbf{X}=\left\{\mathbf{x}_{1}, \ldots, \mathbf{x}_{N}\right\}$ and $\mathbf{Y}=\left\{\mathbf{y}_{1}, \ldots, \mathbf{y}_{M}\right\}$, where $\mathbf{x}_{i}, \mathbf{y}_{i} \in \mathbb{R}^{D}$, algorithm starts by building the local cost matrix $\mathbf{C}$ representing all pairwise distances between $\mathbf{X}$ and $\mathbf{Y}$ :

$$
\mathbf{C} \in \mathbb{R}^{N \times M}: c_{i, j}=\left\|\mathbf{x}_{i}-\mathbf{y}_{j}\right\|, i \in[i: N], j \in[1: M]
$$

Based on the Local Cost Matrix $\mathbf{C}$, we can construct the Accumulated Cost Matrix M, which contains all possible warping paths (see Algorithm 1). Then Dynamic Programming is used to find the optimal warping path and DTW distance, starting from the point $p_{\text {end }}=(M, N)$ to the $p_{\text {start }}=(1,1)$ (Algorithm 2).

To our case, when an anomalous RSSI pattern is detected, we first segment a data stream with $m$ continuous time sample (we choose $m=8$ ), starting from where we detect as an abnormal point. Then, we calculate all the DTW dis-
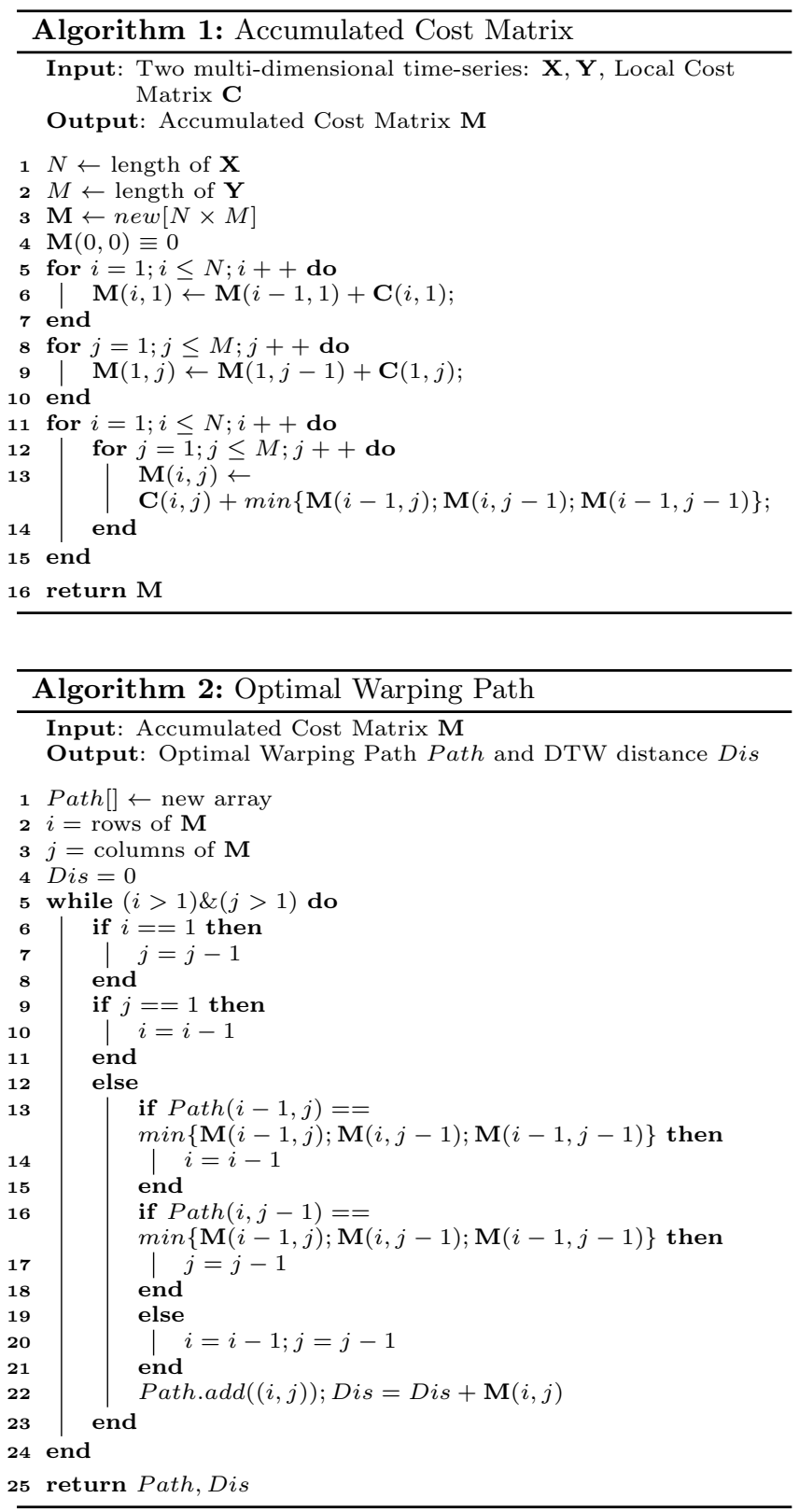

tances between the segmented data stream and the profiling data streams using the multi-dimensional DTW by optimal matching between two given RSSI sequences. Finally, we can classify the falling directions based on a majority voting 


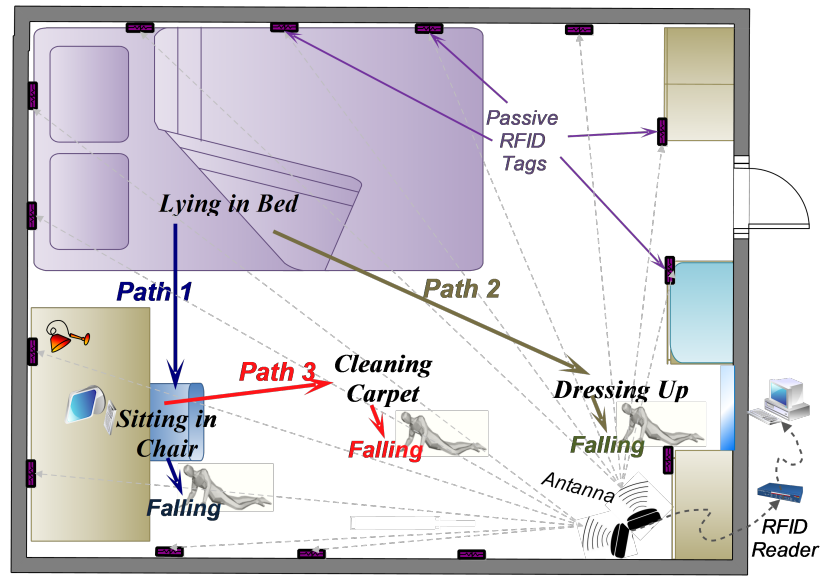

Figure 9: Room layout and three representative action paths

by its top $k$ smallest DTW distances. Figure 8 illustrates the general idea of our DTW based $k \mathrm{NN}$.

\section{EVALUATION}

We evaluate our system in a real-world living bedroom (size: $3.9 m \times 3.6 m$ ). Fig 9 shows the experimental setup and furniture deployment. Two subjects participate in the experiments, one male (Age $: 28$, Height $=172 \mathrm{~cm}$, Weight $=$ $68 \mathrm{~kg}$ ) and one female (Age $: 27$, Height $=163 \mathrm{~cm}$, Weight $=$ $49 \mathrm{~kg})$.

\subsection{Evaluation Metrics}

For regular actions and falling direction classification, we use standard precision, recall and accuracy to measure our proposed approaches [23]. For fall detection, we evaluate our result in terms of Detect Rate and False Detect Rate [12].

$$
\begin{gathered}
\text { DetectRate }=\frac{\text { True Positive }}{\# \text { of Fall Events }} \\
\text { FalseRate }=\frac{\text { False Positive }}{\# \text { of nonFall Events }}
\end{gathered}
$$

\subsection{Sensing Normal Activities and Falls}

We first collect our profiling data, which involves normal daily living activities (see Figure 10, time span is one day). Then we mimic overall 20 different fall events, including various falling directions and locations, shown by 11. All fall events are conducted by both participants repeating 3 times each (i.e., 120 fall events).

Based on the collected profiling data, we first calculate the angle variance of RSSI vectors paired by same action category (i.e., sitting, standing, lying and walking, illustrated by Figure 7 (a) $\sim(d))$. The mean value of variances for regular actions is ranged from $5.14 \times 10^{-14}$ to $4.73 \times 10^{-12}$, but the maximum value of angle variance paired by falling to regular actions (shown by Figure 7 (e)) is $1.36 \times 10^{-14}$. Thus, we can easily separate the space of regular actions with falls. Figure 12 shows our predefined regular activity categories and the learned variance boundaries. We set the lower and upper bound of box diagram as $15 \%$ and $85 \%$, so the interquartile range includes $70 \%$ of most possible variances. From the box

\begin{tabular}{|l|l|}
\hline \multicolumn{1}{|c|}{ Locations } & \multicolumn{1}{c|}{ Activities } \\
\hline Lying in Bed & play mobilephone, read books, listen music \\
\hline Siting in Bed & play mobilephone, play laptop, read books \\
\hline Siting in Chair & paly computer, read books, drink water, writting \\
\hline Standing in Front of Mirror & dress up \\
\hline Standing beside Bed & read books, stretch body \\
\hline Standing beside Desk & paly computer, read books \\
\hline Standing in Front of Wardrobe & take out clothes, put in clothes \\
\hline Standing in Center of Bedroom & do morning exercise \\
\hline Walking Clean Carpet & clean carpet \\
\hline Walking Enter 1 & walk from door to bed \\
\hline Walking Enter 2 & walk from door to chair \\
\hline Walking Enter 3 & walk from door to the front of mirror \\
\hline Walking Out 1 & walk from bed to door \\
\hline Walking Out 2 & walk from chair to door \\
\hline Walking Out 3 & walk from the front of mirror to door \\
\hline Walking Random in Bedroom & \\
\hline
\end{tabular}

Figure 10: Types of normal activities

\begin{tabular}{|l|c|}
\hline \multicolumn{1}{|c|}{ Fall Location } & Fall Direction \\
\hline Falling from Bed 1 & \\
\hline Falling from Bed 2 & \\
\hline Falling from Bed 3 & Right \\
\hline Falling from Chair & Left \\
\hline Falling from Chair & Front \\
\hline Falling from Standing in Center of Room & Back \\
\hline Falling from Standing in Center of Room & Left \\
\hline Falling from Standing in Center of Room & Right \\
\hline Falling from Standing in Center of Room & Left \\
\hline Falling from Standing in Front of Mirror & Right \\
\hline Falling from Standing in Front of Mirror & Back \\
\hline Falling from Standing in Front of Mirror & Left \\
\hline Falling from Standing in Front of Wardrobe & Right \\
\hline Falling from Standing in Front of Wardrobe & Back \\
\hline Falling from Standing in Front of Wardrobe & Front \\
\hline Falling while Cleaning Carpet & Back \\
\hline Falling while Cleaning Carpet & Right \\
\hline Falling while Cleaning Carpet & Left \\
\hline Falling while Cleaning Carpet & \\
\hline Falling while Enter the Room & \\
\hline
\end{tabular}

Figure 11: Different falls in the experiments

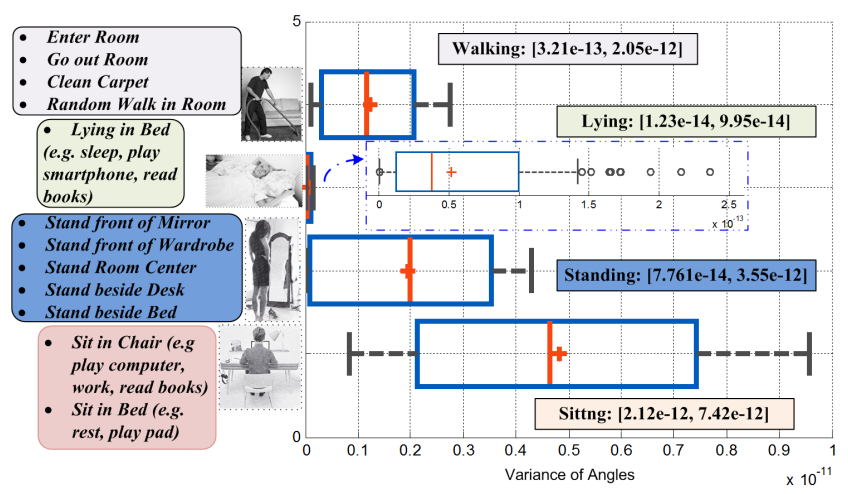

Figure 12: Regular activity categories and boundaries

and whisker diagrams, we can easily determine the variance range for each action. The boundaries of regular actions are ranged from $7.42 \times 10^{-14}$ to $1.23 \times 10^{-12}$, in which the lowest value of all four lower boundaries (i.e., $7.42 \times 10^{-14}$ ) is bigger than the maximum value of angle variance calculated by falling to regular actions (i.e., $1.36 \times 10^{-14}$ ). This further 


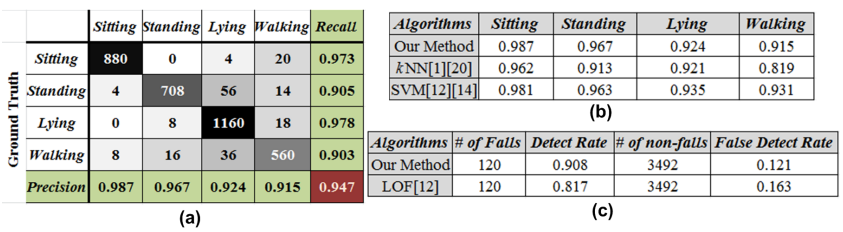

Figure 13: Confusion Matrix and Detection Performance

verifies the feasibility of our method.

After the boundaries for each normal action are learned, we collect 3,492 non-fall events (varies in time length) generated by regular activities to test our method (e.g., reading book in bed, cleaning carpet, see Figure 10), which can achieve overall 94.7\% accuracy. Figure 13 (a) (b) illustrate the performance of sensing regular activities. $k \mathrm{NN}$ and SVM are two classification methods that are frequently used by other fall detection systems [23]. Thus, we compare our method to $k \mathrm{NN}[1,20](k=5)$ and SVM [12, 14] (linear kernel, termination criterion $=0.015, \mathrm{C}=100$, others as default [7]). Our method performs well in distinguishing sitting (98.7\% accuracy) and standing (96.7\% accuracy) actions but slightly worse in lying (92.4\% accuracy) and walking (91.5\% accuracy). $k \mathrm{NN}$ method only achieves $81.9 \%$ in classifying walking action. Our method does not require tuning any parameters and achieves comparable good accuracy, although SVM performs slightly better in distinguishing walking (93.1\% accuracy) and Lying (93.5\% accuracy) action.

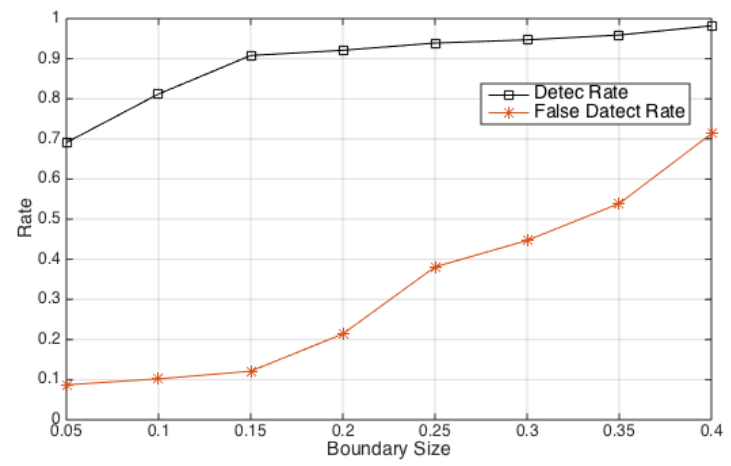

Figure 14: Detection rate and false detection rate varies with the boundaries size ( $\mathrm{X}$-axis only shows the lower boundary, so upper boundary should be $100 \%$ - LowerBoundary, the boundary range should be UpperBoundary - LowerBoundary)

Figure 13 shows the capability of our method in detecting falls. We test overall 120 fall events, including falls from working at desk, dressing up, cleaning the carpet, and falling to different orientations (e.g., falling to front, to back, to right and to left, shown by Figure 11) and 3,492 nonfall events. The result shows that our method can achieve $90.8 \%$ detection rate and $12.1 \%$ false detection rate. As a comparison, we also utilize LOF (adopted by WiFall [12]) to our dataset, which receives a $81.7 \%$ detection rate and $16.3 \%$ false detection rate. In this setting, we set the boundaries of box diagram (Figure 12) as from $15 \%$ to $85 \%$. We can choose different boundaries of the box diagram (e.g., $5 \% \sim 95 \%, 10 \% \sim 90 \%, 20 \% \sim 80 \%$, see Figure 14). It illustrates that both the detection rate and false detection rate increase when the boundary size becomes smaller (spans from $90 \%$ to $20 \%$ ). However, the false detection rate experiences dramatic growth but the true detection rate in fact does not significantly increase (from $90.8 \%$ at $15 \%$ to $98.2 \%$ at $40 \%$ ). Thus, we choose $15 \%$ and $85 \%$ as our lower and upper boundaries in term of the box and whisper diagram.

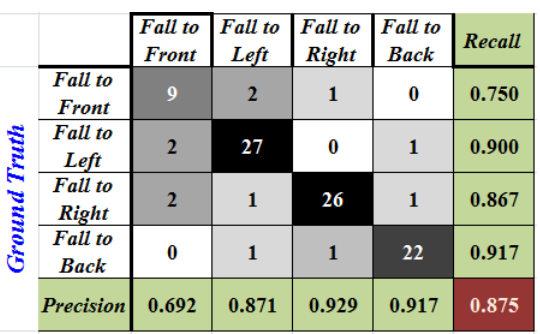

Figure 15: Confusion Matrix of DTW based $k$ NN $(k=3)$

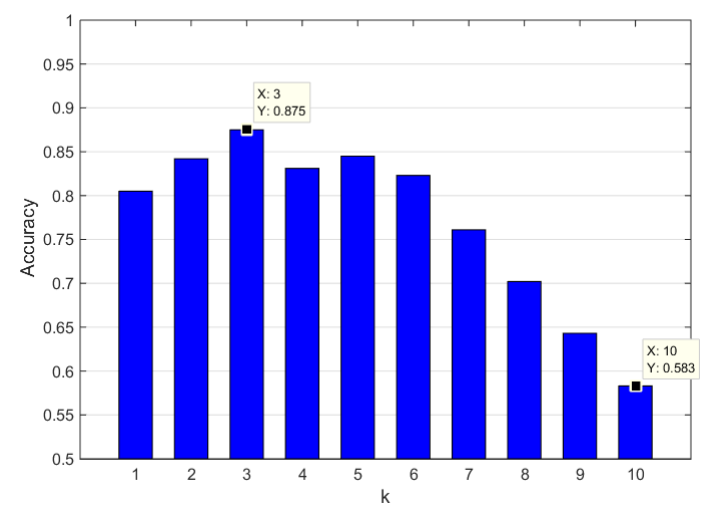

Figure 16: Accuracy of classifying falling direction varies with parameter $k$

For falling direction classification, we choose 16 fall events which contain falling directions (see Figure 11, some falls have no direction context such as falling from bed). Each fall is conducted by both participants and repeated 3 times each (overall 96 fall events). Figure 15 shows the confusion matrix of our DTW based $k \mathrm{NN}$ method choosing $k=3$. We can observe that the overall accuracy is $87.5 \%$, but the precision and recall in classifying falling to front are only $69.2 \%$ and $75 \%$. The reason may lie in fact that falling to front and falling to right/left are quite similar in some cases since these two falling directions are adjacent. Our method performs good at distinguishing the falling to back (precision and recall are both $91.7 \%$ ) which possible cause severe damage to the head. The key parameter in DTW based $k \mathrm{NN}$ is the $k$ value that heavily affects the classification accuracy. Figure 16 illustrates the relation of classifying accuracy with parameter $k$. As it shows, the accuracy at first increases with the growth of $k$ value, climbing the peak at $k=3$, then gradually decreases along with the increase of $k$. Thus, we choose $k=3$ in our experiments.

Figure 17 shows performance of our system in three representative action paths. The bold lines are the ground truth, 


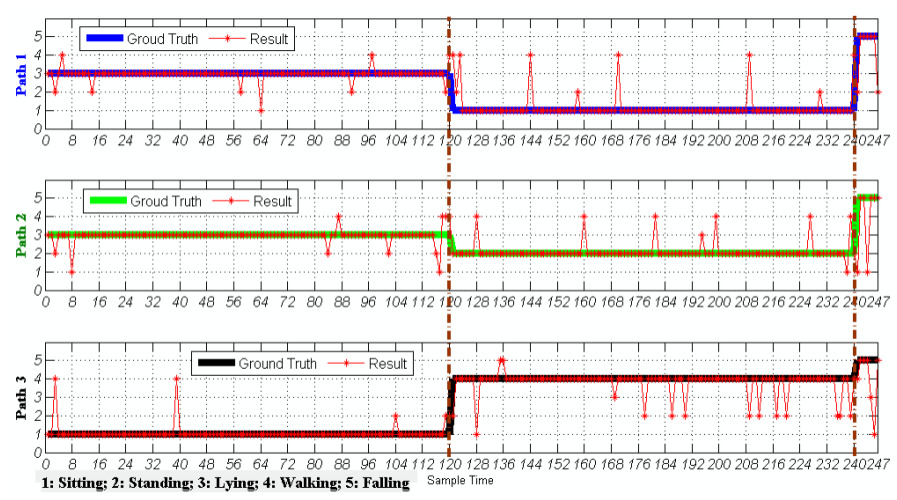

Figure 17: Detect fall events in action paths

the $\mathrm{Y}$-axis from 1 to 5 represent action categories (i.e., sitting, standing, lying, walking and falling). The three action paths are shown by Figure 9:

- Lying in Bed (60 seconds) $\Longrightarrow$ Sitting in Chair (60 seconds) $\Longrightarrow$ Falling Down,

- Lying in Bed (60 seconds) $\Longrightarrow$ Dressing Up in Front of Mirror (60 seconds) $\Longrightarrow$ Falling Down,

- Sitting in Chair (60 seconds) $\Longrightarrow$ Cleaning the Carpet (60 seconds) $\Longrightarrow$ Falling Down.

We can see that in the first action path, our method can timely distinguish the actions and detect the fall event, although generating some unstable predictions when the resident transfers from getting up from bed to sitting in chair. Our system on the second action path displays the same classifying capability, but it outputs some bad predictions after the resident falls from dressing up although it successfully detects a fall event in the first few points. From the third action path, we observe that the classification result is not as good as previous two paths when the subject is cleaning the carpet, for the reason that cleaning involves plenty of activities that may generate some similar RSSI patterns as sitting and standing actions. In summary, when an activity shift occurs (e.g., from lying to sitting in the chair, from sitting to cleaning the carpet), the sensing results are usually decayed, which is normal due to unpredictable movements of human body. After detecting a fall, the continuing sensing result is unstable since people usually lie or sit on ground after a fall, which is similar to our predefined regular activities.

\section{RELATED WORK}

Timely detecting a fall event can abbreviate the damage degree and reduce the morality for the elderly. Fall detection for elderly has been a hot topic in health-care industry and has attracted a lot of attention from academia in the past two decades. Since early 1990s, many fall detection systems have been proposed by researchers from different communities. In [23, 25], the hardware and methods used in existing fall detection systems have been thoroughly discussed and reviewed. Based on the hardware used by fall detection, current systems can be classified into four groups: wearable sensor based, smart-phone based, vision-based and environmental sensor based techniques. From the point of

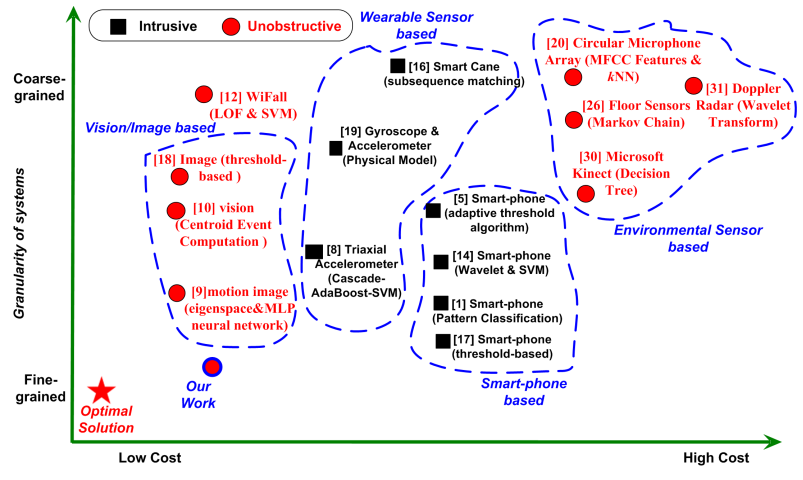

Figure 18: Design Space: comparing to related work

obstructiveness, the former two categories can be regarded as device-free, the latter two are of intrusive in general.

Wearable sensor based fall detection systems rely on sensors that are embedded in wearable stuff such as coat, belt and watch or be taken by hand, such as smart cane. The widely used sensors include inertial sensors [17], tri-axial accelerometers [8], gyroscopes [19] and smart cane [16]. Lee et al. [17] proposed a novel vertical velocity-based fall detection method to detect a fall event using a wearable inertial sensor. Cheng et al. [8] designed a cascade-AdaBoost-SVM classifier to realize a real-time fall detection method based on tri-axial accelerometers worn on the body. Li et al. [19] presented a fall detection system using both accelerometers and gyroscopes, in which linear acceleration and angular velocity are measured to determine whether motion transitions are intentional. In [16], Lan et al. present and design an automatic fall detection system by using a smart cane. These detection systems can only work on the premise that all the devices are worn by the subject and connected correctly to the human body. Such requirements give additional burden and interfere subjects' daily life, which are impractical for some applications.

Most modern smart-phones have built-in sensors that can measure motion, orientation, and various environmental conditions. These sensors are capable of providing raw data with high precision and accuracy. Thus, smart phone based fall detection is promising and with good potential [1], which can integrate all sensors into one single mobile device (e.g., inertial sensors [2], tri-axial accelerometers [5] and gyroscopes [14]). However, smart-phone based fall detection systems share the same mechanism as wearable sensors based techniques. They also have the same problem with wearable based methods. Most users may not take with their phones all the time, especially at home.

Much work has also been done in investigating the use of standard imaging sensors for fall detection. Approaches have ranged from single cameras mounted on the wall to multiple cameras placed around a room [10,9], or to using a depthcamera Kinect [30, 21]. Lee [18] detected a fall by analyzing the shape and $2 \mathrm{D}$ velocity of the person. Rougier [27] used wall-mounted cameras to cover large areas and falls were detected using human shape variation. Despite the considerable achievements that have accomplished in this field over the recent years, traditional camera-based systems still suffer from a number of limitations. The problem this method brings is that people may feel uncomfortable with a camera overhead, especially in bathroom. Besides the privacy in- 
trusion, this method is also limited by line of sight problem and fails in darkness, where falls usually happen.

Device-free fall detection that use environmental sensors attempt to fuse ambient noise information including thermal distribution [13], audio [20], floor vibrational [26], Channel State Information (CSI) [12] data and microwave signal [31] produced by a fall for the detection purpose. The principle is based on the fact that human movements in a living setting will cause the signal variations of environmental sensors (e.g., pressure senors [26], acoustic sensors [20], thermal sensors [13] and wireless transceivers [12], radars [31]), which can be regarded as being less intrusive. For example, WiFall [12] employs the time variability and special diversity of Channel State Information (CSI) as the indicator of human activities to infer a fall event. However, current device-free fall detection systems focus more on detecting a fall event in some predefined areas and fail to provide finegrained information such as status before falling and fall orientations, which may be valuable for rescuers. Figure 18 illustrates our device-free, fine-grained fall detection system based on pure UHF passive RFID tags in the design space of current FD systems. Compared to other hardware platforms, RFID is cost-effective (passive tags cost several cents each) and practical (e.g., no maintenance needs, no battery) and promising in identifying environmental changes [24, 28]. In the meantime, our FD system can provide fine-grained contextual information of a fall event, including what is people doing before falls and the falling orientation.

\section{DISCUSSION}

Computation Cost: In the Profile Construction phase (offline part), for a daily recorded activities, based our configuration, the calculation time for angle variances is around 70 minutes. With the constructed profile data, we can online process each given data sample (i.e., the fall detection phase) within 0.4 seconds. For the falling direction sensing phase, the calculation complexity of DTW is $O(N M)$ [11] (both equal to 8 in our case), so calculation itself is fast. However, we need to segment a fix-length data stream beforehand, which results in a latency (about 4 seconds in our case). However, in the direction sensing phase, we aim to provide fine-grained contexts regarding the happened falls, which does not affect timely detecting a fall and sending an alarm (done in the fall detection phase).

Hardware: We use standard, commercial RFID system with passive tags in our work. The passive tags are more costeffective and, due to their simple structure and protective encapsulation, more robust than the sensor nodes. Passive tags operate without batteries. Once deployed, no further maintenance is required. The devices that require power in our sensing system is the RFID reader and antenna. But recent technical trends show that low-cost, low-power RFID readers are becoming commonly available by integrating into the smart phones, making our work potentially beneficial to the more users in the future.

The Methods: As for fall detection techniques, current fall detection systems mainly adopt supervised classificationbased method to detect a fall event, such as Support Vector Machine (SVM) [2, 8, 12], Neural Network [9] or Extreme Learning Machine [21], which have to tune plenty of parameters to achieve satisfied accuracy. But in our fall detection phase, we aim to mine the clustering patterns of RSSIs based on the variances of angle paired by data point of different actions when the environment is affected by diverse human activities. Thus, different to the traditional classification or distance-based anomaly detection methods, our proposed method relaxes the requirement of tuning parameters that is time-consuming and sensitive to different test scenarios [6].

The Limitations: One of limitations is that the current system is designed for and tested with only a single resident. We believe that this is an important use case, particularly in an aging-in-place setting, which aims to ensure that a single person can live in his/her home and community safely and independently regardless of age and ability level. However, the number of profiles needed with multiple persons would increase exponentially. A more promising approach therefore would be to find techniques that can isolate concurrent activities in separate space from each other and match them against profiles separately, which we will consider in our future work. Another limitation is that labeling profiling data is time-consuming and labor-intensive, which is also an issue shared by other fall detection systems. In the Profile Construction phase, we have to use a camera to record the daily living activities, and then synchronize the camera and RSSI reading based on the time stamp, finally label and segment data streams into different action categories to build a labeled profile dataset based on the video records.

\section{CONCLUSION}

To detect a fall event in our daily living environments, we present an unobstructive, fine-grained fall detection system based on pure passive RFID tags. By proposing a $p$-partially Angle-based Outlier Detection method, our system can simultaneously identify regular activities and detect a fall event. By adopting DTW-based $k \mathrm{NN}$, the proposed system can distinguish different falling orientations. Our approach relaxes the requirement of tuning parameters and provides more fine-grained contexts regarding fall events comparing to the current fall detection systems. In the future, we will enhance our system in detecting falls of multiple subjects and making the system more robust.

\section{REFERENCES}

[1] S. Abbate, M. Avvenuti, F. Bonatesta, G. Cola, P. Corsini, and A. Vecchio. A smartphone-based fall detection system. Pervasive and Mobile Computing, 8(6):883 - 899, 2012.

[2] O. Aziz, C. Russell, E. Park, and S. Robinovitch. The effect of window size and lead time on pre-impact fall detection accuracy using support vector machine analysis of waist mounted inertial sensor data. In Engineering in Medicine and Biology Society $(E M B C), 2014$ 36th Annual International Conference of the IEEE, pages 30-33, Aug 2014.

[3] F. Bianchi, S. Redmond, M. Narayanan, S. Cerutti, and N. Lovell. Barometric pressure and triaxial accelerometry-based falls event detection. Neural Systems and Rehabilitation Engineering, IEEE Transactions on, 18(6):619-627, Dec 2010.

[4] M. M. Breunig, H.-P. Kriegel, R. T. Ng, and J. Sander. Lof: Identifying density-based local outliers. In Proc. of the ACM SIGMOD, 2000. 
[5] Y. Cao, Y. Yang, and W. Liu. E-falld: A fall detection system using android-based smartphone. In Fuzzy Systems and Knowledge Discovery (FSKD), 2012 9th International Conference on, pages 1509-1513, May 2012.

[6] V. Chandola, A. Banerjee, and V. Kumar. Anomaly detection: A survey. ACM Comput. Surv., 41(3):15:1-15:58, 2009.

[7] C.-C. Chang and C.-J. Lin. Libsvm: A library for support vector machines. ACM Trans. Intell. Syst. Technol., 2(3):27:1-27:27, May 2011.

[8] W.-C. Cheng and D.-M. Jhan. Triaxial accelerometer-based fall detection method using a self-constructing cascade-adaboost-svm classifier. Biomedical and Health Informatics, IEEE Journal of, 17(2):411-419, March 2013.

[9] H. Foroughi, A. Naseri, A. Saberi, and H. Yazdi. An eigenspace-based approach for human fall detection using integrated time motion image and neural network. In Signal Processing, 2008. ICSP 2008. 9th International Conference on, pages 1499-1503, Oct 2008.

[10] Z. Fu, E. Culurciello, P. Lichtsteiner, and T. Delbruck. Fall detection using an address-event temporal contrast vision sensor. In Circuits and Systems, 2008. ISCAS 2008. IEEE International Symposium on, May 2008.

[11] N. Gillian, R. B. Knapp, and S. OâĂŹModhrain. Recognition of multivariate temporal musical gestures using n-dimensional dynamic time warping. In Proc of the 11th Int'l Conf. on New Interfaces for Musical Expression, 2011.

[12] C. Han, K. Wu, Y. Wang, and L. Ni. Wifall: Device-free fall detection by wireless networks. In INFOCOM, 2014 Proceedings, April 2014.

[13] P. Hevesi, S. Wille, G. Pirkl, N. Wehn, and P. Lukowicz. Monitoring household activities and user location with a cheap, unobtrusive thermal sensor array. In Proceedings of the 2014 ACM International Joint Conference on Pervasive and Ubiquitous Computing, UbiComp '14, pages 141-145, 2014.

[14] L.-J. Kau and C.-S. Chen. A smart phone-based pocket fall accident detection, positioning, and rescue system. Biomedical and Health Informatics, IEEE Journal of, 19(1):44-56, Jan 2015.

[15] H.-P. Kriegel, M. S hubert, and A. Zimek. Angle-based outlier detection in high-dimensional data. In Proc. of the 14th ACM SIGKDD, 2008

[16] M. Lan, A. Nahapetian, A. Vahdatpour, L. Au, W. Kaiser, and M. Sarrafzadeh. Smartfall: An automatic fall detection system based on subsequence matching for the smartcane. In Proceedings of the Fourth International Conference on Body Area Networks (BodyNets '09), pages 8:1-8:8, 2009.

[17] J. Lee, S. Robinovitch, and E. Park. Inertial sensing-based pre-impact detection of falls involving near-fall scenarios. Neural Systems and Rehabilitation Engineering, IEEE Transactions on, 23(2):258-266, March 2015.

[18] T. Lee and A. Mihailidis. An intelligent emergency response system: preliminary development and testing of automated fall detection. Journal of telemedicine and telecare, 11(4):194-198, 2005.

[19] Q. Li, J. Stankovic, M. Hanson, A. Barth, J. Lach, and G. Zhou. Accurate, fast fall detection using gyroscopes and accelerometer-derived posture information. In Wearable and Implantable Body Sensor Networks, 2009. BSN 2009. Sixth International Workshop on, pages 138-143, June 2009.

[20] Y. Li, K. Ho, and M. Popescu. A microphone array system for automatic fall detection. Biomedical Engineering, IEEE Transactions on, 59(5):1291-1301, May 2012.

[21] X. Ma, H. Wang, B. Xue, M. Zhou, B. Ji, and Y. Li. Depth-based human fall detection via shape features and improved extreme learning machine. Biomedical and Health Informatics, IEEE Journal of, 18(6):1915-1922, Nov 2014.

[22] N. Mitton and D. Simplot-Ryl. Is rfid dangerous?, 2011, http://www.inria.fr/en/centre/lille/news/is-rfiddangerous.

[23] M. Mubashir, L. Shao, and L. Seed. A survey on fall detection: Principles and approaches. Neurocomputing, 100(0):144 - 152, 2013.

[24] L. Ni, D. Zhang, and M. Souryal. Rfid-based localization and tracking technologies. Wireless Communications, IEEE, 18(2):45-51, April 2011.

[25] N. Noury, A. Fleury, P. Rumeau, A. Bourke, G. Laighin, V. Rialle, and J. Lundy. Fall detection principles and methods. In Engineering in Medicine and Biology Society, EMBS 2007, 29th Annual International Conference of the IEEE, pages 1663-1666, 2007.

[26] H. Rimminen, J. Lindstrom, M. Linnavuo, and R. Sepponen. Detection of falls among the elderly by a floor sensor using the electric near field. Information Technology in Biomedicine, IEEE Transactions on, 14(6):1475-1476, Nov 2010.

[27] C. Rougier, J. Meunier, A. St-Arnaud, and J. Rousseau. Fall detection from human shape and motion history using video surveillance. In Advanced Information Networking and Applications Workshops, 2007, AINAW'0\%. 21st International Conference on, volume 2, pages 875-880. IEEE, 2007.

[28] W. Ruan, L. Yao, Q. Z. Sheng, X. Li, and N. J. Falkner. Tagtrack: Device-free localization and tracking using passive rfid tags. In Proc. of the 11th Intl. Conf. on Mobile and Ubiquitous Systems: Computing, Networking and Services, MOBIQUITOUS '14, 2014.

[29] A. Sixsmith and N. Johnson. A smart sensor to detect the falls of the elderly. Pervasive Computing, IEEE, 3(2):42-47, April 2004.

[30] E. Stone and M. Skubic. Fall detection in homes of older adults using the microsoft kinect. Biomedical and Health Informatics, IEEE Journal of, 19(1):290-301, Jan 2015.

[31] B. Su, K. Ho, M. Rantz, and M. Skubic. Doppler radar fall activity detection using the wavelet transform. Biomedical Engineering, IEEE Transactions on, 62(3):865-875, March 2015.

[32] R. Tideiksaar. Falls in older people: Prevention and management. Health Professions Press, 2002. 